\title{
Optimal Reward on a Sparse Tree with Random Edge-Weights
}

\author{
Davar Khoshnevisan* Thomas M. Lewis \\ University of Utah Furman University
}

May 21, 2002

\begin{abstract}
It is well known that the maximal displacement of a random walk indexed by an $m$-ary tree with bounded i.i.d. edge-weights can reliably yield much larger asymptotics than a classical random walk whose summands are drawn from the same distribution. Presently we show that if the edge-weights are mean-zero, then nonclassical asymptotics arise even when the tree grows much more slowly than exponentially. Our conditions are stated in terms of a Minkowski-type logarithmic dimension of the boundary of the tree.
\end{abstract}

Keywords Optimal reward, tree-indexed random walks, logarithmic dimension

AMS 2000 subject classification $60 \mathrm{G} 50,05 \mathrm{C} 05$

\section{Introduction}

Let $T$ be a locally-finite infinite rooted tree with vertex set $\mathcal{V}$, edge set $\mathcal{E}$, and root $\mathfrak{r}$. We will associate with each edge $\mathfrak{e} \in \mathcal{E}$ a signed weight $\omega_{\mathfrak{e}}$, that we think of as the reward for selecting that edge. Given a path in $T$, the reward for selecting the path is the cumulative sum of the weights of the edges that compose the path. An optimal path of depth $n$ is a path with maximal reward from the set of all rooted paths of length $n$; the optimal reward at depth $n$ denoted by $M_{n}(\omega)$-is the reward for this optimal path. In this paper, we study the effect of the thickness of the tree on the asymptotic value of $M_{n}(\omega)$.

We will assume that the edge-weights $\omega=\left\{\omega_{\mathfrak{e}} ; \mathfrak{e} \in \mathcal{E}\right\}$ are a collection of real i.i.d. random variables; that is, given any real Borel probability measure $\mathbb{P}, \omega$ is a random variable under the product measure $\mathbb{P}^{\mathcal{E}}$, which is a Borel probability measure on the product space $\mathbb{R}^{\mathcal{E}}$. We will assume, furthermore, that

$$
\forall \mathfrak{e} \in \mathcal{E}, \mathbb{E}\left\{\omega_{\mathfrak{e}}\right\}=0, \mathbb{E}\left\{\omega_{\mathfrak{e}}^{2}\right\}=1, \text { and } \exists \lambda_{0}>0, \mathbb{E}\left\{e^{\lambda_{0}\left|\omega_{\mathfrak{e}}\right|}\right\}<+\infty
$$

${ }^{*}$ Research partially supported by grants from the NSF and NATO 
These are more or less the usual moment conditions on the edge-weights. In Section 4 we will show that the conditions of (1.1) are essentially optimal in the setting of this paper.

We will need some additional notation for trees. An ordered collection (finite or infinite) $\sigma=\mathfrak{v}_{0} \mathfrak{v}_{1} \mathfrak{v}_{2} \ldots$ of distinct vertices is called a path provided that there is an edge between $\mathfrak{v}_{i-1}$ and $\mathfrak{v}_{i}, i \geq 1$; the path $\sigma$ is said to originate at $\mathfrak{v}_{0}$. Equivalently, a path can be described by a sequence of adjacent edges $\sigma=\left(\mathfrak{v}_{0}, \mathfrak{v}_{1}\right)\left(\mathfrak{v}_{1}, \mathfrak{v}_{2}\right) \ldots$

Given two paths $\sigma_{1}$ and $\sigma_{2}$, we will write $\sigma_{1} \prec \sigma_{2}$ provided that they have a common origin, and each vertex of $\sigma_{1}$ is a vertex of $\sigma_{2}$. In other words, $\sigma_{2}$ is an extension of $\sigma_{1}$. If the path $\sigma$ is composed of a finite number of vertices, then the length of $\sigma$-denoted by $|\sigma|$-is the number of adjacent edges composing the path; otherwise, $|\sigma|=+\infty$.

By a ray we mean a path that originates at the root $\mathfrak{r}$. For completeness, we will enlarge the set of rays by attaching the empty ray, a ray composed of no edges and having zero length. Given two rays $\sigma$ and $\sigma^{\prime}$, let $\sigma \curlywedge \sigma^{\prime}$ denote the ray of maximal length such that $\sigma \curlywedge \sigma^{\prime} \prec \sigma$ and $\sigma \curlywedge \sigma^{\prime} \prec \sigma^{\prime}$. Let $\partial T_{n}$ and $\partial T$ denote respectively the set of all rays of length $n$ and the set of all infinite rays in $T$. Moreover, the number of rays of length $n$ is denoted by $A(n)=A_{n}=\left|\partial T_{n}\right|$. Hereafter, we will assume that to each $\sigma \in \partial T_{n}$ there exits $\widehat{\sigma} \in \partial T$ such that $\sigma \prec \widehat{\sigma}$. In other words, each vertex of $T$ is part of an infinite ray. It follows from this that $A_{1} \leq A_{2} \leq \cdots$. With one exception (Theorem 3.1), we will assume that $|\partial T|=+\infty$.

Given $\sigma=\mathfrak{r} \mathfrak{v}_{1} \mathfrak{v}_{2} \ldots \in \partial T$, let $S_{0}^{\sigma}=0$, and for $n \geq 1$ let

$$
S_{n}^{\sigma}=\omega_{\left(\mathfrak{r}, \mathfrak{v}_{1}\right)}+\omega_{\left(\mathfrak{v}_{1}, \mathfrak{v}_{2}\right)}+\cdots+\omega_{\left(\mathfrak{v}_{n-1}, \mathfrak{v}_{n}\right)} .
$$

The stochastic process $\left\{S_{n}^{\sigma}: n \geq 0, \sigma \in \partial T\right\}$ is called a $T$-indexed random walk. The notation $S_{n}^{\sigma}$ can be unambiguously extended to rays $\sigma$ of finite length provided that $n \leq|\sigma|$, and hereafter we will use this convention. In this notation, the optimal reward at depth $n$ can be expressed as

$$
M_{n}(\omega)=\max \left\{S_{n}^{\sigma}(\omega) ; \sigma \in \partial T_{n}\right\} .
$$

In a slightly different context, BIGGins (1977) has shown that whenever $T$ grows exponentially, then $\lim _{n \rightarrow \infty} \frac{1}{n} M_{n}(\omega)$ exists for $\mathbb{P}^{\mathcal{E}}$-almost all $\omega$, and depends on the entire distribution $\mathbb{P}$. Earlier works can be found in Dubins and Freedman (1967), Hammersley (1974), Kingman (1975), and related results can be found in Aldous (1992), Benjamini And PeRes (1994), Lyons And Pemantle (1992), and Peres (1999). Among other things, these works investigate trees where $M_{n}$ is of order $n$.

Presently, we are concerned with the value of the optimal reward on sparse trees; that is, trees with a subexponential branching rate (see equation 1.5). We were motivated by the following questions:

(1) What features of $T$ guarantee the existence of $a_{n} \rightarrow \infty$ such that

$$
\lim _{n \rightarrow \infty} \frac{M_{n}(\omega)}{a_{n}}=1, \quad \text { for } \mathbb{P}^{\mathcal{E}} \text {-almost all } \omega \in \mathbb{R}^{\mathcal{E}} \text { ? }
$$


(2) For what sparse trees can we find $a_{n} \rightarrow \infty$ with the property that

$$
0<\liminf _{n \rightarrow \infty} \frac{M_{n}(\omega)}{a_{n}} \leq \limsup _{n \rightarrow \infty} \frac{M_{n}(\omega)}{a_{n}}<+\infty
$$

for $\mathbb{P}^{\mathcal{E}}$-almost all $\omega \in \mathbb{R}^{\mathcal{E}}$ ?

$\left(3\right.$ For what sparse trees can we find $a_{n} \rightarrow \infty$ with the property that

$$
\liminf _{n \rightarrow \infty} \frac{M_{n}(\omega)}{a_{n}} \leq 0<\limsup _{n \rightarrow \infty} \frac{M_{n}(\omega)}{a_{n}}<+\infty
$$

for $\mathbb{P}^{\mathcal{E}}$-almost all $\omega \in \mathbb{R}^{\mathcal{E}}$ ?

In words, 1 asks when the optimal reward has a limiting value; asks when there is a reliable, though oscillatory, asymptotic value for this reward; finally, $\mathbf{3}$ asks when the asymptotic value of this reward is unreliable in that it fluctuates wildly as we move down the tree.

Our approach will be to characterize the growth of $M_{n}$ via geometric conditions on $T$. In this sense we are close, in spirit, to the studies of BENJAMINI And Peres (1994) and Lyons And Pemantle (1992) for large trees. Recently VIRÁG (2001) has investigated a set of problems closely related to those of this present article.

It is difficult to answer Questions (1-3 in complete generality; however, if the tree satisfies some mild regularity conditions, then some general answers do emerge. To this end, the following function is fundamental to our analysis: For each $\lambda \geq 1$, let

$$
\varrho(\lambda)=\limsup _{n \rightarrow \infty} \frac{\ln A(\lfloor\lambda n\rfloor)}{\ln A(n)} .
$$

We say that $T$ is log-regular provided that this $\lim \sup$ is a limit for all $\lambda \geq 1$, and we say that $T$ is thin provided that $\varrho(\lambda)=1$ for every $\lambda \geq 1$.

Remark 1.1 It is clear that $\varrho(\lambda) \geq 1$ for $\lambda \geq 1$, and that $\varrho$ is monotone increasing in $\lambda$. For $a, b \geq 1$, observe that $\varrho(a b) \leq \varrho(a) \varrho(b)$; thus, if $\varrho(\lambda)<\infty$ for some $\lambda>1$, then $\varrho(\lambda)<\infty$ for every $\lambda \geq 1$. If $T$ is log-regular, then $\varrho(a b)=\varrho(a) \varrho(b)$ for $a, b \geq 1$. In particular, if $\varrho(2)$ is finite, then $\varrho\left(2^{\frac{1}{n}}\right)=$ $\exp \left\{\frac{1}{n} \ln [\varrho(2)]\right\}$ for all $n \geq 1$, and consequently, $\varrho(\lambda) \downarrow 1$ as $\lambda \downarrow 1$.

Hereafter, we will tacitly restrict our attention to the class of trees that satisfy the following sparseness condition:

$$
\lim _{n \rightarrow \infty} \frac{1}{n} \ln A_{n}=0, \text { and } \varrho(2)<+\infty .
$$

The first assumption will be used to carry out a moderate deviations estimate. We note that this assumption can be removed whenever the edge-weights are normally distributed. By Remark 1.1, $\varrho(2)<+\infty$ is equivalent to $\varrho(\lambda)<+\infty$ for all $\lambda \geq 1$. 
Finally we introduce some notation for measuring the rate of growth of $T$. Let

$$
\overline{\operatorname{dim}}_{\mathcal{L}}(\partial T)=\limsup _{n \rightarrow \infty} \frac{\ln A_{n}}{\mathrm{~L}_{2} n}, \quad \underline{\operatorname{dim}}_{\mathcal{L}}(\partial T)=\liminf _{n \rightarrow \infty} \frac{\ln A_{n}}{\mathrm{~L}_{2} n} .
$$

These are the upper logarithmic dimension and the lower logarithmic dimension of the boundary of $T$ respectively, where

$$
\mathrm{L}_{2} x=\ln _{+} \ln _{+} x, \quad \forall x \geq 0,
$$

and $\ln _{+} x=\max (\ln x, 1)$. When the upper and lower logarithmic dimensions are equal, we will denote their common value by $\operatorname{dim}_{\mathcal{L}}(\partial T)$. Quite naturally, we call this the logarithmic dimension of the boundary of $T$.

We have a variety of answers to Questions (1) - (3) hold under different regularity conditions on $T$. However, our results are most elegant and complete when $(i) T$ is thin, and $(i i) \operatorname{dim}_{\mathcal{L}}(\partial T)$ exists. In such a case, we find:

(4) If $\operatorname{dim}_{\mathcal{L}}(\partial T)=+\infty$, then $\lim _{n \rightarrow \infty}\left(2 n \ln A_{n}\right)^{-\frac{1}{2}} M_{n}(\omega)=1$, for $\mathbb{P}^{\mathcal{E}}$-almost all $\omega \in \mathbb{R}^{\mathcal{E}}$; see Corollary 3.5 below.

(5) On the other hand, if $\operatorname{dim}_{\mathcal{L}}(\partial T)<+\infty$, then for $\mathbb{P}^{\mathcal{E}}$-almost all $\omega \in \mathbb{R}^{\mathcal{E}}$,

$$
\liminf _{n \rightarrow \infty} \frac{M_{n}(\omega)}{\sqrt{2 n \mathrm{~L}_{2} n}}=\sqrt{\operatorname{dim}_{\mathcal{L}}(\partial T)}
$$

whereas

$$
\limsup _{n \rightarrow \infty} \frac{M_{n}(\omega)}{\sqrt{2 n \mathrm{~L}_{2} n}}=\sqrt{1+\operatorname{dim}_{\mathcal{L}}(\partial T)}
$$

see Corollaries 3.4 and 3.5 below.

Thus, for such trees $T$,

$$
\begin{aligned}
& \text { (1) } \Longleftrightarrow \operatorname{dim}_{\mathcal{L}}(\partial T)=+\infty, \\
& \text { (2) } \Longleftrightarrow 0<\operatorname{dim}_{\mathcal{L}}(\partial T)<+\infty, \\
& \text { (3) } \Longleftrightarrow \operatorname{dim}_{\mathcal{L}}(\partial T)=0 .
\end{aligned}
$$

The preceding discussion shows that when $d=\operatorname{dim}_{\mathcal{L}}(\partial T)<+\infty, \mathbb{P}^{\mathcal{E}}$-every realization of $n \mapsto\left(2 n \mathrm{~L}_{2} n\right)^{-\frac{1}{2}} M_{n}$ clusters inside $[\sqrt{d}, \sqrt{d+1}]$. It would be interesting to know if the latter interval is in fact the entire cluster set. Quite generally, this is the case. Indeed, we have the following.

Theorem 1.2 Under the sparseness condition (1.5), and if $d=\operatorname{dim}_{\mathcal{L}}(\partial T)$ is finite but $\partial T$ is infinite, then

$$
\liminf _{n \rightarrow \infty} \sup _{x \in[\sqrt{d}, \sqrt{d+1}]}\left|\frac{M_{n}}{\sqrt{2 n f(n)}}-x\right|=0,
$$

$\mathbb{P}^{\mathcal{E}}$-almost surely. 
Viewing $T$ as a subexponential decision tree, each ray $\sigma \in \partial T$ can be interpreted as a strategy. In light of the preceding discussion, it is natural to wish to find a near-optimal strategy; that is, one may wish to find a ray $\sigma \in \partial T$ such that $S_{n}^{\sigma}$ has the same growth rate as $M_{n}$ as $n$ increases without bound. In Section 5.1 we will show that, for spherically symmetric trees, there is a kind of hit-or-miss near-optimal strategy, and in Section 6.1 we will show that consistent near-optimal strategies do not exist for thin spherically symmetric trees. See Theorems 5.1 and 6.1 for precise statements of these results.

Acknowledgement A portion of this work was completed while one of us (D. Kh.) was visiting Ecole Polytechnique Fédérale de Lausanne. We wish to thank EPF-L for their hospitality. We also thank Amir Dembo for providing us with references on moderate deviations.

\section{The Law of the Iterated Logarithm}

We will begin our work by analyzing the largest possible value of the optimal reward. We recall that equations (1.1) and (1.5) are in effect.

Theorem 2.1 (The Law of the Iterated Logarithm) There exists a constant $C_{2.1} \in(0, \infty)$ such that for $\mathbb{P}^{\mathcal{E}}$-almost all $\omega \in \mathbb{R}^{\mathcal{E}}$,

$$
\limsup _{n \rightarrow \infty} \frac{M_{n}(\omega)}{\sqrt{2 n\left[\ln A_{n}+\mathrm{L}_{2} n\right]}}=C_{2.1}, \quad \mathbb{P}^{\mathcal{E}} \text {-a.s. }
$$

If the tree is log-regular, then $C_{2.1} \leq 1$; if the tree is thin, then $C_{2.1}=1$.

For this, we will need the following form of a moderate deviations result; see Dembo And Zeitouni (1998, p. 109), for example. For the sake of completeness, we have included an Appendix A, where the reader can find an alternative (and elementary) derivation of moderate deviations that is based on the classical central limit theorem.

Lemma 2.2 (Moderate Deviations) Suppose $V_{1}, V_{2}, \ldots$ are random i.i.d. meanzero variance-one random variables that satisfy the following integrability condition for some $\lambda_{0}>0: \mathbb{E}\left\{\exp \left(\lambda_{0}\left|V_{1}\right|\right)\right\}<+\infty$. Then, given an unbounded sequence $x_{n}=o(n)$,

$$
\mathbb{P}\left\{Y_{n} \geq \sqrt{n x_{n}}\right\}=\exp \left\{-\frac{1}{2}(1+o(1)) x_{n}\right\} \quad(n \rightarrow \infty),
$$

where $Y_{n}$ denotes either $\sum_{j=1}^{n} V_{j}$ or $\max _{\ell \leq n} \sum_{j=1}^{\ell} V_{j}$. 
Proof of Theorem 2.1 For simplicity, let

$$
f(n)=\ln A_{n}+\mathrm{L}_{2} n .
$$

Choose $\lambda, p>1$, and let $n(k)=n_{k}=\left[\lambda^{k}\right]$ for $k \geq 1$. We see at once that

$\mathbb{P}\left\{\max _{j \leq n(k)} M_{j}(\omega) \geq \sqrt{2 p n_{k} f\left(n_{k}\right)}\right\} \leq \sum_{\sigma \in \partial T_{n(k)}} \mathbb{P}\left\{\max _{j \leq n(k)} S_{j}^{\sigma}(\omega) \geq \sqrt{2 p n_{k} f\left(n_{k}\right)}\right\}$.

We can apply Lemma 2.2 with $x_{n}=f(n)$ and deduce that as $k \rightarrow \infty$,

$$
\begin{aligned}
\mathbb{P}\left\{\max _{j \leq n(k)} S_{j}^{\sigma}(\omega) \geq \sqrt{2 p n_{k} f\left(n_{k}\right)}\right\} & =\exp \left\{-(1+o(1)) p f\left(n_{k}\right)\right\} \\
& =A_{n(k)}^{-p+o(1)} k^{-p+o(1)}
\end{aligned}
$$

Since $A_{n(k)}=\left|\partial T_{n(k)}\right|$,

$$
\mathbb{P}\left\{\max _{j \leq n(k)} M_{j}(\omega) \geq \sqrt{2 p n_{k} f\left(n_{k}\right)}\right\} \leq k^{-p+o(1)},
$$

as $k \rightarrow \infty$. By the Borel-Cantelli lemma, eventually,

$$
\max _{j \leq n(k)} M_{j}(\omega)<\sqrt{2 p n_{k} f\left(n_{k}\right)},
$$

on an $\omega$-set of full $\mathbb{P}^{\mathcal{E}}$-measure. Since $p>1$ is arbitrary and, by (1.4),

$$
\limsup _{k \rightarrow \infty} \frac{n_{k+1} f\left(n_{k+1}\right)}{n_{k} f\left(n_{k}\right)} \leq \lambda \varrho(\lambda)
$$

it is evident that

$$
\limsup _{n \rightarrow \infty} \frac{M_{n}(\omega)}{\sqrt{2 n f(n)}} \leq \sqrt{\lambda \varrho(\lambda)}, \quad \mathbb{P}^{\mathcal{E}} \text {-a.s. }
$$

The right-hand side may be replaced by $\lim _{\lambda \downarrow 1} \sqrt{\varrho(\lambda)}$, yielding a universal upper bound. If, moreover, the tree is log-regular, then $\varrho(\lambda) \rightarrow 1$ as $\lambda \rightarrow 1^{+}$; thus, in the presence of log-regularity, the upper bound can be taken as 1 .

We now argue toward a lower bound. Choose $p>1, q \in(0,1), \lambda>2$, and let $n_{k}=n(k)=\left[\lambda^{k}\right], k \geq 1$. For each $\sigma \in \partial T_{n(k)}$, fix one path $\widehat{\sigma} \in \partial T_{n(k+1)}$ such that $\widehat{\sigma} \succ \sigma$, and let

$$
E_{\sigma}=\left\{\omega: S_{n(k+1)}^{\widehat{\sigma}}(\omega)-S_{n(k)}^{\sigma}(\omega) \geq \sqrt{2 q\left(n_{k+1}-n_{k}\right) f\left(n_{k}\right)}\right\} .
$$

By Lemma 2.2, as $k \rightarrow \infty$,

$$
\mathbb{P}\left\{E_{\sigma}\right\}=\exp \left\{-(1+o(1)) q f\left(n_{k}\right)\right\} \geq \frac{C_{\lambda}}{k A_{n(k)}},
$$


where $C_{\lambda}>0$ depends only on $\lambda$. Consequently,

$$
\begin{array}{rlrl}
\mathbb{P}\left\{\bigcup_{\sigma \in \partial T_{n(k)}} E_{\sigma}\right\} & =1-\mathbb{P}\left\{\bigcap_{\sigma \in \partial T_{n(k)}} E_{\sigma}^{\mathrm{C}}\right\} & \\
& =1-\left[1-\mathbb{P}\left(E_{\gamma}\right)\right]^{A_{n(k)}} & & \left(\forall \gamma \in \partial T_{n(k)}\right) \\
& \geq 1-\exp \left\{-A_{n(k)} \mathbb{P}\left(E_{\gamma}\right)\right\} & & \left(\forall \gamma \in \partial T_{n(k)}\right) \\
& \geq \frac{C_{\lambda}}{2 k},
\end{array}
$$

for all sufficiently large $k$. By the Borel-Cantelli lemma for independent events, $\mathbb{P}^{\mathcal{E}}$-almost surely there are infinitely many indices $k$ for which there exist paths $\sigma \in \partial T_{n(k)}$ and $\widehat{\sigma} \in \partial T_{n(k+1)}$ with $\sigma \prec \widehat{\sigma}$ such that

$$
S_{n(k+1)}^{\widehat{\sigma}}(\omega)-S_{n(k)}^{\sigma}(\omega) \geq \sqrt{2 q\left(n_{k+1}-n_{k}\right) f\left(n_{k}\right)} .
$$

Recalling (2.2), it follows that there exist infinitely many $k$ such that

$$
\begin{aligned}
& M_{n(k+1)}(\omega) \\
& \quad \geq \sqrt{2 q\left(n_{k+1}-n_{k}\right) f\left(n_{k}\right)}-\sqrt{2 p n_{k} f\left(n_{k}\right)} \\
& \quad \geq \sqrt{2 n_{k+1} f\left(n_{k+1}\right)} \sqrt{\frac{f\left(n_{k}\right)}{f\left(n_{k+1}\right)}} \times\left[\sqrt{q\left(1-\frac{n_{k}}{n_{k+1}}\right)}-\sqrt{p\left(\frac{n_{k}}{n_{k+1}}\right)}\right],
\end{aligned}
$$

on a set of full $\mathbb{P}^{\mathcal{E}}$ measure. Since $p>1$ and $q \in(0,1)$ are arbitrary, it is evident that

$$
\limsup _{n \rightarrow \infty} \frac{M_{n}(\omega)}{\sqrt{2 n f(n)}} \geq \frac{1}{\sqrt{\varrho(\lambda)}}\left[\sqrt{1-\frac{1}{\lambda}}-\frac{1}{\sqrt{\lambda}}\right], \quad \mathbb{P}^{\mathcal{E}} \text {-a.s. }
$$

The right-hand side is positive, which, by the zero-one law, gives the general result. If $T$ is thin, that is $\varrho(\lambda)=1$ for each $\lambda \geq 1$, then by letting $\lambda$ increase without bound we see that the bound on the right-hand side can be replaced by 1 , as asserted.

\section{A Law of Large Numbers}

In this section we present some results concerning the slowest possible growth rates for the value of the optimal reward. The fastest possible growth rates have been discussed in Theorem 2.1, where it was shown that under mild conditions on the tree $T$,

$$
\limsup _{n \rightarrow \infty} \frac{M_{n}(\omega)}{\sqrt{2 n\left(\ln A_{n}+\mathrm{L}_{2} n\right)}}=C_{2.1}, \quad \mathbb{P}^{\mathcal{E}} \text {-a.s. }
$$

While the greater part of this section is devoted to three general results on the slowest growth of the optimal reward, our ultimate goal is to verify 
the corollaries that follow. In essence, we show that if a tree has a positive but finite logarithmic dimension, then the optimal reward will be ultimately positive, but will oscillate (Corollary 3.4); however, if the tree has infinite logarithmic dimension, then the optimal reward satisfies a strong law of large numbers (Corollary 3.5).

Our first result is a general theorem concerning the limit inferior of the optimal reward.

Theorem 3.1 For $\mathbb{P}^{\mathcal{E}}$-almost all $\omega \in \mathbb{R}^{\mathcal{E}}$,

$$
\liminf _{n \rightarrow \infty} \frac{M_{n}(\omega)}{\sqrt{2 n \mathrm{~L}_{2} n}} \begin{cases}=-|\partial T|^{-1 / 2}, & \text { if }|\partial T|<\infty, \\ \geq 0, & \text { if }|\partial T|=\infty .\end{cases}
$$

The second result of this section presents an upper bound on the limit inferior in terms of the upper logarithmic dimension.

Theorem 3.2 If $\overline{\operatorname{dim}}_{\mathcal{L}}(\partial T)<+\infty$, then

$$
\liminf _{n \rightarrow \infty} \frac{M_{n}(\omega)}{\sqrt{2 n\left(\ln A_{n}+\mathrm{L}_{2} n\right)}} \leq \sqrt{\frac{\overline{\operatorname{dim}}_{\mathcal{L}}(\partial T)}{\operatorname{dim}_{\mathcal{L}}(\partial T)+1}}, \quad \mathbb{P}^{\mathcal{E}} \text {-a.s. }
$$

If $T$ is log-regular and $\overline{\operatorname{dim}}_{\mathcal{L}}(\partial T)=\infty$, then the result holds with the convention that $\infty \div \infty=1$.

Our next result gives information about the limit inferior in terms of the lower logarithmic dimension.

Theorem 3.3 If $\underline{\operatorname{dim}}_{\mathcal{L}}(T)>0$, then

$$
\liminf _{n \rightarrow \infty} \frac{M_{n}(\omega)}{\sqrt{2 n\left(\ln A_{n}+\mathrm{L}_{2} n\right)}}=C_{3.3}>0, \quad \mathbb{P}^{\mathcal{E}} \text {-a.s. }
$$

If, in addition, $T$ is thin, then

$$
C_{3.3} \geq \sqrt{\frac{\underline{\operatorname{dim}}_{\mathcal{L}}(\partial T)}{1+\underline{\operatorname{dim}}_{\mathcal{L}}(\partial T)}},
$$

where $\infty \div \infty=1$.

The following corollaries follows directly from Theorems $2.1,3.1,3.2$, and 3.3 .

Corollary 3.4 If $|\partial T|=+\infty$ and $\operatorname{dim}_{\mathcal{L}}(\partial T)<+\infty$, then almost surely,

$$
\liminf _{n \rightarrow \infty} \frac{M_{n}}{\sqrt{2 n \mathrm{~L}_{2} n}}=\sqrt{\operatorname{dim}_{\mathcal{L}}(\partial T)}, \quad \text { and } \quad \limsup _{n \rightarrow \infty} \frac{M_{n}}{\sqrt{2 n \mathrm{~L}_{2} n}}=\sqrt{1+\operatorname{dim}_{\mathcal{L}}(\partial T)} \text {. }
$$

Corollary 3.5 (Law of Large Numbers) Suppose that $T$ is thin and $\operatorname{dim}_{\mathcal{L}}(\partial T)=$ $+\infty$. Then,

$$
\lim _{n \rightarrow \infty} \frac{M_{n}}{\sqrt{2 n \ln A_{n}}}=1, \quad \mathbb{P}^{\mathcal{E}} \text {-a.s. }
$$


Proof of Theorem 3.1 Let us first assume that $N=|\partial T|<+\infty$, and let

$$
\gamma=\max \left\{\left|\sigma \curlywedge \sigma^{\prime}\right|: \sigma, \sigma^{\prime} \in \partial T\right\} .
$$

The key observation is that below level $\gamma$, the tree $T$ has no further branching; thus, our random walk on $T$ ultimately behaves like $N$ independent random walks.

Let $\alpha$ be chosen such that $N \alpha>1$. Choose $\lambda>1$ and consider the sequence $n_{k}=n(k)=\left\lfloor\lambda^{k}\right\rfloor$. For $\sigma \in \partial T$ and $n(k) \geq \gamma$, let

$$
H_{k}^{\sigma}=\left\{\omega: \min _{\gamma \leq j \leq n(k)}\left(S_{j}^{\sigma}(\omega)-S_{\gamma}^{\sigma}(\omega)\right) \leq-\sqrt{2 \alpha\left(n_{k}-\gamma\right) \mathrm{L}_{2} n_{k}}\right\} .
$$

Due to the definition of $\gamma$, the events $\left\{H_{k}^{\sigma}: \sigma \in \partial T\right\}$ are independent. Thus, by the moderate deviations estimate (Lemma 2.2) and the fact that $A\left(n_{k}\right)=N$ for $n_{k} \geq \gamma$, it is evident that

$$
\begin{aligned}
q_{k}(\alpha) & =\mathbb{P}\left\{\bigcap_{\sigma_{i} \in \partial T} H_{k}^{\sigma}\right\} \\
& =\left(\exp \left\{-(1+o(1)) \alpha \mathrm{L}_{2} n_{k}\right\}\right)^{A\left(n_{k}\right)} \\
& \leq k^{-N(\alpha+o(1))} .
\end{aligned}
$$

Since $N \alpha>1$, the sequence $\left\{q_{k}(\alpha)\right\}_{k \geq 1}$ sums in $k$; thus, by the Borel-Cantelli lemma, there exists a set of full $\mathbb{P}^{\mathcal{E}}$ measure on which, eventually,

$$
\min _{\gamma \leq j \leq n_{k}}\left(S_{j}^{\sigma}(\omega)-S_{\gamma}^{\sigma}(\omega)\right)>-\sqrt{2 \alpha\left(n_{k}-\gamma\right) \mathrm{L}_{2} n_{k}},
$$

for some $\sigma \in \partial T$. Thus, $\mathbb{P}^{\mathcal{E}}$ almost surely, given $n_{k}<n \leq n_{k+1}$ sufficiently large,

$$
M_{n}(\omega) \geq-\sqrt{2 \alpha\left(n_{k+1}-\gamma\right) \mathrm{L}_{2} n_{k+1}}-\max _{\sigma \in \partial T}\left(-S_{\gamma}^{\sigma}\right),
$$

and it follows that

$$
\liminf _{n \rightarrow \infty} \frac{M_{n}(\omega)}{\sqrt{2 n \mathrm{~L}_{2} n}} \geq-\lambda \sqrt{\alpha}, \quad \mathbb{P}^{\mathcal{E}} \text {-a.s. }
$$

We conclude this part of the proof by letting $\lambda$ decrease to 1 and $\alpha$ decrease to $N^{-1}=|\partial T|^{-1}$ along rational sequences.

Next let us assume that $\alpha>0$ and $N \alpha<1$ and consider the events

$$
G_{k}^{\sigma}=\left\{\omega: S_{n(k+1)}^{\sigma}-S_{n(k)}^{\sigma} \leq-\sqrt{2 \alpha\left(n_{k+1}-n_{k}\right) \mathrm{L}_{2} n_{k+1}}\right\} .
$$

By moderate deviations (Lemma 2.2), for $n_{k} \geq \gamma$,

$$
\begin{aligned}
p_{k}(\alpha) & =\mathbb{P}\left\{\bigcap_{\sigma \in \partial T_{n(k+1)}} G_{k}^{\sigma}\right\} \\
& =\left(\exp \left\{-(1+o(1)) \alpha \mathrm{L}_{2} n_{k+1}\right\}\right)^{N} \\
& \geq k^{-N \alpha+o(1)} .
\end{aligned}
$$


Since $N \alpha<1$, the sequence $\left\{p_{k}(\alpha)\right\}_{k \geq 1}$ has a divergent sum. Thus, by the Borel-Cantelli lemma, there exists a set of full measure on which

$$
S_{n(k+1)}^{\sigma} \leq-\sqrt{2 \alpha\left(n_{k+1}-n_{k}\right) \mathrm{L}_{2} n_{k+1}}+S_{n(k)}^{\sigma},
$$

for each $\sigma \in \partial T_{n(k+1)}$, for infinitely many indices $n_{k}$. Thus, for almost every $\omega$,

$$
\frac{M_{n(k+1)}}{\sqrt{2 n_{k+1} \mathrm{~L}_{2} n_{k+1}}} \leq-\sqrt{\alpha} \sqrt{1-\frac{n_{k}}{n_{k+1}}}+\frac{M_{n(k)}}{\sqrt{2 n_{k} \mathrm{~L}_{2} n_{k}}} \sqrt{\frac{n_{k}}{n_{k+1}}},
$$

for infinitely many indices $n_{k}$. Thus, by Theorem 2.1 ,

$$
\liminf _{n \rightarrow \infty} \frac{M_{n}}{\sqrt{2 n \mathrm{~L}_{2} n}} \leq-\sqrt{\alpha} \sqrt{1-\frac{1}{\lambda}}+\frac{C_{2.1}}{\sqrt{\lambda}}, \quad \mathbb{P}^{\mathcal{E}} \text {-a.s. }
$$

We achieve the desired conclusion upon letting $\lambda$ increase without bound, and letting $\alpha$ increase to $\frac{1}{N}$ along rational sequences. This concludes our proof in the case where $T$ has finite boundary.

If $|\partial T|=\infty$, let $M \geq 1$ be chosen and let $\sigma_{1}, \sigma_{2}, \ldots, \sigma_{M}$ be $M$ rays in $\partial T$. Let $T^{\prime}$ denote the sub-tree of $T$ with $\partial T^{\prime}=\left\{\sigma_{1}, \sigma_{2}, \ldots, \sigma_{M}\right\}$ and let $\omega^{\prime}=\left\{\omega_{\mathfrak{e}} ; \mathfrak{e} \in \mathcal{E}^{\prime}\right\}$, where $\mathcal{E}^{\prime} \subset \mathcal{E}$ is the induced edge-set of $T^{\prime}$. Now it is clear from this construction that $M_{n}(\omega) \geq M_{n}\left(\omega^{\prime}\right)$; thus, by our result for trees with finite boundary,

$$
\liminf _{n \rightarrow \infty} \frac{M_{n}(\omega)}{\sqrt{2 n \mathrm{~L}_{2} n}} \geq \liminf _{n \rightarrow \infty} \frac{M_{n}\left(\omega^{\prime}\right)}{\sqrt{2 n \mathrm{~L}_{2} n}}=-\frac{1}{\sqrt{M}}, \quad \mathbb{P}^{\mathcal{E}} \text {-a.s. }
$$

Since this is true for each $M \geq 1$, the result follows.

Proof of Theorem 3.2 For simplicity, let $d=\overline{\operatorname{dim}}_{\mathcal{L}}(\partial T)$. If $T$ is log-regular and $d=+\infty$, then the result follows trivially from Theorem 2.1. Otherwise, $d<\infty$. In this case, we note that

$$
\ln A_{n} \leq(d+o(1)) \mathrm{L}_{2} n .
$$

Choose $\alpha>d /(d+1)$, observing that $\alpha$ satisfies

$$
(1-\alpha) d-\alpha<0 \text {. }
$$

Choose $\lambda>1, n_{k}=n(k)=\left\lfloor\lambda^{k}\right\rfloor, k \geq 1$, and let

$$
\varepsilon_{k}=\sqrt{2 \alpha\left(n_{k+1}-n_{k}\right) f\left(n_{k+1}\right)} .
$$

Since random walks are associated, (ESARY ET AL. 1967),

$$
\begin{aligned}
p_{k}(\alpha) & =\mathbb{P}\left(\bigcap_{\sigma \in \partial T_{n(k+1)}}\left\{S_{n(k+1)}^{\sigma}(\omega)-S_{n(k)}^{\sigma}(\omega) \leq \varepsilon_{k}\right\}\right) \\
& \geq \prod_{\sigma \in \partial T_{n(k+1)}} \mathbb{P}\left\{S_{n(k+1)}^{\sigma}-S_{n(k)}^{\sigma} \leq \varepsilon_{k}\right\} \\
& =\left(1-\mathbb{P}\left\{S_{n(k+1)-n(k)}^{\sigma}>\varepsilon_{k}\right\}\right)^{A_{n(k+1)}} .
\end{aligned}
$$


On the other hand, moderate deviations and (3.1) together show that

$$
\begin{aligned}
A_{n(k+1)} \mathbb{P}\left\{S_{n(k+1)-n(k)}>\varepsilon_{k}\right\} & =\exp \left(-(1+o(1)) \alpha f\left(n_{k+1}\right)+\ln A_{n(k+1)}\right) \\
& =\exp \left(\mathrm{L}_{2} n_{k}((1-\alpha) d-\alpha+o(1))\right) .
\end{aligned}
$$

Consequently, equations (3.2) and (3.3) together show that the probabilities $\left\{p_{k}(\alpha)\right\}_{k \geq 1}$ are bounded away from zero. By the Borel-Cantelli lemma for independent events, there exists a set of full measure on which

$$
S_{n(k+1)} \leq \varepsilon_{k}+S_{n(k)}, \quad \forall \sigma \in \partial T_{n(k)},
$$

for infinitely many indices $n_{k}$. This implies that almost surely

$$
M_{n(k+1)} \leq \varepsilon_{k}+M_{n(k)},
$$

for infinitely many indices $n_{k}$. Recalling the definition of $\varepsilon_{k}$, it is evident that almost surely

$$
\frac{M_{n(k+1)}(\omega)}{\sqrt{2 n_{k+1} f\left(n_{k+1}\right)}} \leq \sqrt{\alpha} \sqrt{1-\frac{n_{k}}{n_{k+1}}}+\frac{M_{n(k)}(\omega)}{\sqrt{2 n_{k} f\left(n_{k}\right)}} \sqrt{\frac{n_{k}}{n_{k+1}}},
$$

for infinitely many indices $n_{k}$. By Theorem 2.1 we may conclude that

$$
\liminf _{n \rightarrow \infty} \frac{M_{n}}{\sqrt{2 n f(n)}} \leq \sqrt{\alpha} \sqrt{1-\frac{1}{\lambda}}+\frac{C_{2.1}}{\sqrt{\lambda}}, \quad \mathbb{P}^{\mathcal{E}} \text {-a.s. }
$$

We obtain the final result upon letting $\lambda$ increase without bound and $\alpha$ decrease to $d /(d+1)$ along rational sequences.

Our proof of Theorem 3.3 will require several preparatory lemmas. Given $\varepsilon \in(0,1)$, let

$$
\langle n\rangle(\varepsilon)=\min \left\{j: A_{j} \geq A_{n}^{1-\varepsilon}\right\} .
$$

It follows that $(1-\varepsilon) \ln A_{n} \leq \ln A_{\langle n\rangle(\varepsilon)}$ for each $n$. Our next lemma gives us a convenient upper bound on $\ln A_{\langle n\rangle(\varepsilon)}$.

Lemma 3.6 For each $\varepsilon \in(0,1)$, there exists a positive integer $n_{0}$ such that for all $n \geq n_{0}$,

$$
(1-\varepsilon) \ln A_{n} \leq \ln A_{\langle n\rangle(\varepsilon)} \leq(1-\varepsilon) \varrho(2) \ln A_{n} .
$$

Proof The inequality on the left follows from the definition of $\langle n\rangle(\varepsilon)$. To obtain the inequality on the right, let $c \in\left(\frac{1}{2}, 1\right)$. In accord with the definition of $\varrho$, there exists a positive integer $n_{0}$ such that for all $n \geq n_{0}$,

$$
\ln A_{\langle n\rangle(\varepsilon)} \leq \varrho(2) \ln A_{c\langle n\rangle(\varepsilon)} .
$$

However, since $c\langle n\rangle(\varepsilon)<\langle n\rangle(\varepsilon)$,

$$
\ln A_{c\langle n\rangle(\varepsilon)}<(1-\varepsilon) \ln A_{n},
$$

which has the desired effect. 
Remark 3.7 If $T$ is log-regular, the above proof could be modified to show that

$$
\lim _{n \rightarrow \infty} \frac{\ln A_{\langle n\rangle(\varepsilon)}}{\ln A_{n}}=1 .
$$

We will not make use of this fact in the sequel.

Our next lemma considers the ratio of $\langle n\rangle(\varepsilon)$ to $n$.

Lemma 3.8 For any $\varepsilon \in\left(1-\frac{1}{\varrho(\lambda)}, 1\right)$, there exists a positive integer $n_{0}$, such that for all $n \geq n_{0}$,

$$
\frac{\langle n\rangle(\varepsilon)}{n} \leq \frac{1}{\lambda} .
$$

Proof Given such an $\varepsilon$, note that $\varrho(\lambda)<(1-\varepsilon)^{-1}$; hence, in accord with the definition of $\varrho$, there exists a positive integer $n_{0}$ such that $n \geq n_{0}$ implies

$$
\frac{\ln A(n)}{\ln A(n /\lfloor\lambda\rfloor)}<(1-\varepsilon)^{-1}
$$

equivalently, $A(n /\lfloor\lambda\rfloor)>A\left(n^{1-\varepsilon}\right)$, which demonstrates that $\langle n\rangle(\varepsilon) \geq n \lambda^{-1}$ for $n \geq n_{0}$, as was to be shown.

Remark 3.9 If $T$ is thin, then $\varrho(\lambda)=1$ for every $\lambda \geq 1$. Thus, for each $\varepsilon \in(0,1)$ and $\lambda \geq 1$, Lemma 3.8 asserts that $\lim _{\sup _{n \rightarrow \infty}} \frac{1}{n}\langle n\rangle(\varepsilon) \leq \lambda^{-1}$. From this, it follows that

$$
\lim _{n \rightarrow \infty} \frac{\langle n\rangle(\varepsilon)}{n}=0,
$$

for all $\varepsilon \in(0,1)$.

We have one final preparatory lemma.

Lemma 3.10 For each $\lambda>1$,

$$
\limsup _{k \rightarrow \infty} \max _{n_{k} \leq j \leq n_{k+1}} \frac{\left|M_{j}(\omega)-M_{n(k)}(\omega)\right|}{\sqrt{2 n_{k+1} f\left(n_{k+1}\right)}} \leq \sqrt{\lambda-1}, \quad \mathbb{P}^{\mathcal{E}} \text {-a.s. },
$$

where $n_{k}=n(k)=\left\lfloor\lambda^{k}\right\rfloor$ for $k \geq 1$.

Proof Let $\lambda>1$ be given and let $\alpha>1$ be chosen. By moderate deviations (Lemma 2.2),

$$
\begin{aligned}
p_{k}(\alpha) & \\
& =\mathbb{P}\left\{\max _{n_{k} \leq j \leq n_{k+1}}\left|M_{j}(\omega)-M_{n(k)}(\omega)\right|>\sqrt{2 \alpha\left(n_{k+1}-n_{k}\right) f\left(n_{k+1}\right)}\right\} \\
& \leq \mathbb{P}\left\{\max _{\sigma \in \partial T_{n(k+1)}} \max _{n_{k} \leq j \leq n_{k+1}}\left|S_{j}^{\sigma}(\omega)-S_{n(k)}^{\sigma}(\omega)\right|>\sqrt{2 \alpha\left(n_{k+1}-n_{k}\right) f\left(n_{k+1}\right)}\right\} \\
& \leq 2 A_{n(k+1)} \exp \left(-(1+o(1)) \alpha f\left(n_{k+1}\right)\right) \\
\leq & k^{-\alpha+o(1)} .
\end{aligned}
$$


Since $\alpha>1$, the sequence $\left\{p_{k}(\alpha)\right\}_{k \geq 1}$ has a convergent sum; thus, by the BorelCantelli lemma and the definition of $n_{k}$,

$$
\limsup _{k \rightarrow \infty} \max _{n_{k} \leq j \leq n_{k+1}} \frac{\left|M_{j}(\omega)-M_{n(k)}(\omega)\right|}{\sqrt{2 n_{k+1} f\left(n_{k+1}\right)}} \leq \sqrt{\alpha(\lambda-1)}, \quad \mathbb{P}^{\mathcal{E}_{\text {-a.s. }}}
$$

To complete the proof, let $\alpha \downarrow 1$ along a rational sequence.

Proof of Theorem 3.3 Fix a number $r>1$ and let $\varepsilon$ satisfy $1-1 / \varrho(r)<\varepsilon<1$. Let $\lambda>1$ be chosen and let $n_{k}=n(k)=\left\lfloor\lambda^{k}\right\rfloor, k \geq 1$. Let $0<d<\underline{\operatorname{dim}}_{\mathcal{L}}(T)$ and observe that there exists a positive integer $n_{0}$ such that

$$
\ln A_{n} \geq d \mathrm{~L}_{2} n
$$

for $n \geq n_{0}$. For future reference, let us note that

$$
f(n)<\left(\frac{d+1}{d}\right) \ln A_{n}, \quad \forall n \geq n_{0} .
$$

Since $d>0$, choose $\alpha$ such that $0<\alpha<(1-\varepsilon) d /(d+1)$, noting, in particular, that

$$
(1-\varepsilon-\alpha) d-\alpha>0 .
$$

For each $k \geq 1$ and $\sigma \in \partial T_{\left\langle n_{k}\right\rangle(\varepsilon)}$, let $\sigma^{\prime} \in \partial T_{n_{k}}$ with $\sigma \prec \sigma^{\prime}$, and let

$$
G_{k}^{\sigma}(\alpha)=\left\{S_{n_{k}}^{\sigma^{\prime}}(\omega)-S_{\left\langle n_{k}\right\rangle(\varepsilon)}^{\sigma}(\omega)<\sqrt{2 \alpha\left(n_{k}-\left\langle n_{k}\right\rangle(\varepsilon)\right) f\left(n_{k}\right)}\right\} .
$$

For each $k \geq 1$, the events $\left\{G_{k}^{\sigma}(\alpha): \sigma \in \partial T_{\left\langle n_{k}\right\rangle(\varepsilon)}\right\}$ are i.i.d. Thus, for any fixed $\pi \in \partial T_{\left\langle n_{k}\right\rangle(\varepsilon)}$,

$$
p_{k}(\alpha)=\mathbb{P}\left\{\bigcap_{\sigma \in \partial T_{\left\langle n_{k}\right\rangle(\varepsilon)}} G_{k}^{\sigma}(\alpha)\right\} \leq \exp \left(-A_{\left\langle n_{k}\right\rangle(\varepsilon)}\left[1-P\left\{G_{k}^{\pi}(\alpha)\right\}\right]\right) .
$$

By the definition of $\left\langle n_{k}\right\rangle(\varepsilon)$, our moderate deviations estimate (Lemma 2.2), and (3.5),

$$
\begin{aligned}
\ln p_{k}(\alpha) & \leq-A_{n_{k}}^{1-\varepsilon} \exp \left\{-(1+o(1)) \alpha f\left(n_{k}\right)\right\} \\
& \leq-\exp \left\{((1-\varepsilon-\alpha) d-\alpha+o(1)) \mathrm{L}_{2} n_{k}\right\}
\end{aligned}
$$

Thanks to (3.7), the exponent is ultimately positive; consequently, the sequence $\left\{p_{k}(\alpha)\right\}_{k \geq 1}$ has a convergent sum. By the Borel-Cantelli Lemma, this shows that on a set of full measure, ultimately,

$$
\frac{M_{n_{k}}(\omega)}{\sqrt{2 n_{k} f\left(n_{k}\right)}} \geq \sqrt{\alpha}\left(1-\frac{\left\langle n_{k}\right\rangle(\varepsilon)}{n_{k}}\right)^{\frac{1}{2}}-\frac{M_{\left\langle n_{k}\right\rangle(\varepsilon)}(-\omega)}{\sqrt{2 n_{k} f\left(n_{k}\right)}} .
$$


From Lemma 3.8, we may conclude that

$$
\liminf _{k \rightarrow \infty} \sqrt{\alpha}\left(1-\frac{\left\langle n_{k}\right\rangle(\varepsilon)}{n_{k}}\right)^{\frac{1}{2}} \geq \sqrt{\alpha\left(1-r^{-1}\right)} .
$$

By Lemma 3.9, (3.6), and some algebra,

$$
\frac{\left\langle n_{k}\right\rangle(\varepsilon) f\left(\left\langle n_{k}\right\rangle(\varepsilon)\right)}{n_{k} f\left(n_{k}\right)} \leq \frac{(d+1)(1-\varepsilon)}{r d} \varrho(2) .
$$

Thus, we can apply Theorem 2.1 to the opposite-signed edge-weights $\left\{-\omega_{\mathfrak{e}} ; \mathfrak{e} \in\right.$ $\mathcal{E}\}$, and deduce that

$$
\limsup _{k \rightarrow \infty} \frac{M_{n_{k}}(-\omega)}{\sqrt{2 n_{k} f\left(n_{k}\right)}} \leq C_{2.1} \sqrt{\frac{(d+1)(1-\varepsilon)}{r d} \varrho(2)} .
$$

From this, (3.9), and (3.10), we may conclude that

$$
\liminf _{k \rightarrow \infty} \frac{M_{n_{k}}(\omega)}{\sqrt{2 n_{k} f\left(n_{k}\right)}} \geq \sqrt{\alpha\left(1-r^{-1}\right)}-C_{2.1} \sqrt{\frac{(d+1)(1-\varepsilon)}{r d} \varrho(2)},
$$

on a set of full measure. We can let $\alpha$ increase (along a rational sequence) to $(1-\varepsilon) d /(d+1)$ and conclude that

$$
\liminf _{k \rightarrow \infty} \frac{M_{n_{k}}(\omega)}{\sqrt{2 n_{k} f\left(n_{k}\right)}} \geq \sqrt{1-\varepsilon}\left\{\sqrt{\frac{(r-1) d}{r(d+1)}}-C_{2.1} \sqrt{\frac{(d+1) \varrho(2)}{r d}}\right\},
$$

on a set of full measure. The key observation is that the term inside of the braces is ultimately positive as $r$ increases without bound. Thus, by the zero-one law, there exists a positive constant $c(\lambda)$ such that

$$
\liminf _{k \rightarrow \infty} \frac{M_{n_{k}}(\omega)}{\sqrt{2 n_{k} f\left(n_{k}\right)}}=c(\lambda), \quad \mathbb{P}^{\mathcal{E}} \text {-a.s. }
$$

By letting $\varepsilon$ decrease to $1-1 / \varrho(r)$, we can conclude that

$$
c(\lambda) \geq \sup _{r>1}\left(\frac{1}{\sqrt{\varrho(r)}} \sqrt{\frac{(r-1) d}{r(d+1)}}-C_{2.1} \sqrt{\frac{(d+1) \varrho(2)}{r d}}\right) .
$$

To finish, let $n_{k} \leq j \leq n_{k+1}$, and note that

$$
M_{j}(\omega) \geq M_{n_{k}}(\omega)-\max _{n_{k} \leq j \leq n_{k+1}}\left|M_{j}(\omega)-M_{n_{k}}(\omega)\right| .
$$

By Lemma 3.10,

$$
\begin{aligned}
\liminf _{j \rightarrow \infty} \frac{M_{j}(\omega)}{\sqrt{2 j f(j)} \geq} & \liminf _{k \rightarrow \infty} \frac{M_{n_{k}}(\omega)}{\sqrt{2 n_{k} f\left(n_{k}\right)}} \sqrt{\frac{n_{k} f\left(n_{k}\right)}{n_{k+1} f\left(n_{k+1}\right)}} \\
& -\limsup _{k \rightarrow \infty} \max _{n_{k} \leq j \leq n_{k+1}} \frac{\left|M_{j}(\omega)-M_{n_{k}}(\omega)\right|}{\sqrt{2 n_{k+1} f\left(n_{k+1}\right)}} \sqrt{\frac{n_{k+1} f\left(n_{k+1}\right)}{n_{k} f\left(n_{k}\right)}} \\
\geq & \frac{c(\lambda)}{\sqrt{\lambda \varrho(\lambda)}}-\sqrt{\lambda(\lambda-1) \varrho(\lambda)}, \quad \mathbb{P}^{\mathcal{E}} \text {-a.s. }
\end{aligned}
$$


Letting $\lambda$ decrease to 1 , along a rational sequence, leads to

$$
\liminf _{j \rightarrow \infty} \frac{M_{j}(\omega)}{\sqrt{2 j f(j)}} \geq \sup _{\lambda>1} \frac{c(\lambda)}{\sqrt{\varrho(\lambda)}}, \quad \mathbb{P}^{\mathcal{E}} \text {-a.s. }
$$

By the zero-one law, we may conclude that the liminf converges almost surely to a positive constant $C_{3.3}$.

If $T$ is thin, then $C_{2.1}=1$ and $\varrho(r)=1$ for $r \geq 1$, and (3.11) assumes the form

$$
c(\lambda) \geq \sup _{r>1}\left\{\sqrt{\frac{(r-1) d}{r(d+1)}}-\sqrt{\frac{(d+1)}{r d}}\right\}=\sqrt{\frac{d}{d+1}} .
$$

We can conclude this proof by letting $d \rightarrow \underline{\operatorname{dim}}_{\mathcal{L}}(T)$ along a rational sequence.

We conclude this section by proving Theorem 1.2.

Proof of Theorem 1.2 By combining Theorem 2.1 and Corollary 3.4, we can deduce that with $\mathbb{P}$-probability one, the sequence $\left\{\eta_{n}\right\}_{n \geq 1}$ clusters inside $[\sqrt{d}, \sqrt{d+1}]$. Our goal is to show that $\left\{\eta_{n}\right\}_{n \geq 1}$ almost surely converges to the said interval. We will appeal to the following deterministic lemma whose simple proof is omitted:

Lemma 3.11 Suppose that $\left\{a_{n}\right\}_{n \geq 1}$ is a sequence of real numbers that clusters inside a finite interval $\mathbb{\mathbb { I }}$, and that $\lim _{n \rightarrow \infty}\left(a_{n+1}-a_{n}\right)=$ 0 . Then, $\left\{a_{n}\right\}_{n \geq 1}$ converges to the interval $\mathbb{I}$.

In light of Lemma 3.11, it suffices to show that $\mathbb{P}$-almost surely,

$$
\lim _{n \rightarrow \infty} \frac{M_{n+1}-M_{n}}{\sqrt{n f(n)}}=0 .
$$

Now $M_{n+1}-M_{n}$ is nonnegative, and is not greater than the maximum edgeweight at level $n+1$. In other words, if $V$ is an edge-weight, for any $\varepsilon>0$ and all $n \geq 1$,

$$
\mathbb{P}\left\{M_{n+1}-M_{n} \geq \varepsilon \sqrt{n f(n)}\right\} \leq A_{n+1} \mathbb{P}\{V \geq \varepsilon \sqrt{n f(n)}\} .
$$

By equation (1.1) and Chebyshev's inequality,

$$
\mathbb{P}\left\{M_{n+1}-M_{n} \geq \varepsilon \sqrt{n f(n)}\right\} \leq \mathbb{E}\left\{e^{\lambda_{0}|V|}\right\} A_{n+1} e^{-\varepsilon \lambda_{0} \sqrt{n f(n)}},
$$

which is summable in $n$, since $A_{n}=n^{d+o(1)}(n \rightarrow \infty)$.

Remark 3.12 Equation (3.12) continues to hold when $\operatorname{dim}_{\mathcal{L}}(\partial T)=+\infty$, or when it does not exist; only equations (1.1) and (1.5) are needed to insure this assertion. In light of the preceding argument, the proof of this is simple: Thanks to (3.13), it suffices to show that as $n \rightarrow \infty, A_{n+1}=\exp \left\{o(1) \sqrt{n \ln A_{n}}\right\}$; but this follows readily from (1.5). 


\section{On the Growth Condition (1.1)}

We have effectively shown that under a mild regularity/sparseness condition on the tree $T$, and when the edge-weights satisfy (1.1), the optimal reward on $T$ has a reliably high value if and only if the boundary of $T$ has positive logarithmic dimension. We now ask,

"Can the exponential tail-condition on the edge-weights be relaxed?"

In this section we argue that such refinements cannot be made in a manner that is essential. Indeed, suppose $V, V_{1}, V_{2}, \ldots$ are i.i.d. random variables, each with the same law as the edge-weights. Let us also suppose that the tails of the edge-weights are truly subexponential in the following sense:

$$
\exists \nu \in(0,1): \liminf _{\lambda \rightarrow \infty} \lambda^{-\nu} \ln \mathbb{P}\{V>\lambda\}>0 .
$$

Lemma 4.1 If the edge-weights have mean zero and variance one, and if their tails satisfy (4.1), then, Theorem 2.1 does not hold.

Proof Let us start with the observation that for any $\theta>0$,

$$
\begin{aligned}
\mathbb{P}\left\{\max _{j \leq A_{n}} V_{j} \leq \theta \sqrt{n \ln A_{n}}\right\} & =\left[1-\mathbb{P}\left\{V>\theta \sqrt{n \ln A_{n}}\right\}\right]^{A_{n}} \\
& \leq \exp \left(-A_{n} \mathbb{P}\left\{V>\theta \sqrt{n \ln A_{n}}\right\}\right) .
\end{aligned}
$$

Thus, thanks to (4.1), we can find a finite constant $c_{1}>0$ and a $\nu \in(0,2)$ such that for all $n \geq 1$,

$$
\ln \mathbb{P}\left\{\max _{j \leq A_{n}} V_{j} \leq \theta \sqrt{n \ln A_{n}}\right\} \leq-A_{n} \exp \left(-c_{1} \theta^{\nu}\left[n \ln A_{n}\right]^{\nu / 2}\right) .
$$

Using this, we will show that Theorem 2.1 fails for a tree $T$ where $A_{n}$ goes to $+\infty$ sufficiently rapidly. Indeed, suppose

$$
\exists \zeta \in\left(\frac{\nu}{2-\nu}, 1\right), c_{2}, c_{3}>0: \forall n \geq 1, \quad c_{2} n^{\zeta} \leq \ln A_{n} \leq c_{3} n^{\zeta} .
$$

Since $\zeta<1$, such a tree satisfies condition (1.5). On the other hand, this and (4.2), together, imply that

$$
\ln \mathbb{P}\left\{\max _{j \leq A_{n}} V_{j} \leq \theta \sqrt{n \ln A_{n}}\right\} \leq-\exp \left(c_{2} n^{\zeta}-c_{1} c_{3}^{\nu / 2} \theta^{\nu} n^{(1+\zeta) \nu / 2}\right) .
$$

Since $\zeta>\frac{\nu}{2-\nu}$, the above sums in $n$. Consequently, using this and the fact that $f(n)=(1+o(1)) \ln A_{n}$, we see that for any $\theta>0$,

$$
\exists n_{0} \geq 1: \forall n \geq n_{0}, \quad \max _{j \leq A_{n}} V_{j} \geq \theta \sqrt{n f(n)}, \quad \mathbb{P}^{\mathcal{E}} \text {-a.s. }
$$


where the sequence $f$ was defined in (2.1). Note that $\max _{j \leq A_{n}} V_{j}$ has the same distribution as the maximum edge-weight at level $n$. We will abuse notation and write $V_{j}$ for the edge-weights at level $n$. If $\left|M_{n-1}\right| \leq \frac{\theta}{2} \sqrt{n f(n)}$, and if $n$ were large enough, $M_{n} \geq \max _{j \leq A_{n}} V_{j}-\left|M_{n-1}\right| \geq \frac{\theta}{2} \sqrt{n f(n)}$. Since $\theta$ is arbitrary, this shows that $\limsup _{n \rightarrow \infty}\left|M_{n}\right| / \sqrt{n f(n)}=+\infty$, a.s.

\section{Existence of Near-Optimal Rays}

Viewing $T$ as a decision tree, we can identify each $\sigma \in \partial T$ as a "decision strategy." Bearing this interpretation in mind, it is natural to ask about the existence of asymptotically optimal strategies. In this section we present a partial result toward this end.

We say that $T$ is spherically symmetric if every vertex at level $n$ branches off to $B_{n}=B(n) \in\{1,2, \ldots\}$ vertices. It should be recognized that $A_{n}=$ $\prod_{\ell=1}^{n} B_{\ell}$, and the growth Condition (1.1) is equivalent to $\sum_{j=1}^{n} \ln B_{j}=o(n)$. Since $\ln B_{j} \geq(\ln 2)^{-1} \mathbf{1}_{(1, \infty)}\left(B_{j}\right)$, we immediately obtain that $\left\{j \geq 1: B_{j}>1\right\}$ has zero density; i.e.,

$$
\lim _{n \rightarrow \infty} \frac{\left|\left\{1 \leq j \leq n: B_{j}>1\right\}\right|}{n}=0 .
$$

We now show that Theorem 2.1 can be achieved along a single ray when $T$ is spherically symmetric.

Theorem 5.1 If $T$ is spherically symmetric, then

$$
\sup _{\sigma \in \partial T} \limsup _{n \rightarrow \infty} \frac{S_{n}^{\sigma}}{\sqrt{2 n f(n)}}>0, \quad \mathbb{P}^{\mathcal{E}} \text {-a.s. }
$$

If, in addition, $T$ is thin, then

$$
\sup _{\sigma \in \partial T} \limsup _{n \rightarrow \infty} \frac{S_{n}^{\sigma}}{\sqrt{2 n f(n)}}=\limsup _{n \rightarrow \infty} \sup _{\sigma \in \partial T} \frac{S_{n}^{\sigma}}{\sqrt{2 n f(n)}}=1, \quad \mathbb{P}^{\mathcal{E}} \text {-a.s. }
$$

Proof Following the terminology of (Benjamini And Peres 1994; Lyons 1990,1992), we consider the Gromov metric,

$$
\operatorname{dist}(\sigma, \gamma)=2^{-|\sigma \curlywedge \gamma|}, \quad \forall \sigma, \gamma \in \partial T .
$$

This defines a metric topology on $\partial T$ that is generated by open balls of the form

$$
\operatorname{Ball}\left(\sigma, 2^{-n}\right)=\{\gamma \in \partial T:|\sigma \curlywedge \gamma|>n\}, \quad \forall \sigma \in \partial T, n=1,2, \ldots
$$

Endowed with this metric, the boundary of $T$ is compact. To see this, observe that $\partial T$ can be covered by $A_{m}$ open balls of diameter $2^{-(m-1)}$, as can be seen from a picture. (Alternatively, look at the descendants of each of the vertices 
at depth $m$ separately.) Since this can be done for any integer $m$, ( $\partial T$, dist) is a totally bounded. It is equally easy to see that ( $\partial T$, dist) is complete.

As before, choose $\lambda>1$, and define $n_{k}=n(k)=\left\lfloor\lambda^{k}\right\rfloor, k=1,2, \ldots$ Let us fix an integer $m$ and some $\tau \in \partial T$, and define $U=\operatorname{Ball}\left(\tau, 2^{-m}\right)$. We can view $U$ as a tree in its own right with boundary $\partial U$. To avoid confusion between $T$ and its subtree $U$, we will append a subscript $U$ when necessary. Notably, $A_{U}(n)$ will denote the number of vertices in the $n$th generation of the tree $U$, and $f_{U}(n)=\ln A_{U}(n)+\mathrm{L}_{2} n$. Since $T$ is spherically symmetric,

$$
A_{U}(n)= \begin{cases}1 & \text { if } n \leq m \\ A_{n} / A_{m+1} & \text { if } n \geq m+1\end{cases}
$$

Consequently,

$$
f_{U}(n)=(1+o(1)) f(n) \quad(n \rightarrow \infty) .
$$

For any $k$, consider the random set $F_{k} \subseteq \partial T$, described by

$$
F_{k}=\left\{\sigma \in \partial T: S_{n(k+1)}^{\sigma}-S_{n(k)}^{\sigma} \geq \sqrt{2 q\left(n_{k+1}-n_{k}\right) f\left(n_{k}\right)}\right\} .
$$

It is easy to see that $F_{k}$ is an open subset of $\partial T$, because whenever $\sigma \in F_{k}$, $\operatorname{Ball}\left(\sigma, 2^{-\ell}\right) \subseteq F_{k}$ for all $\ell$ such that $2^{\ell}>n_{k+1}$.

The results of our labors on the law of the iterated logarithm can be applied to the subtree $U$. In particular, (2.5) and (5.1) together imply that

$$
\mathbb{P}\left\{F_{k} \cap \partial U \neq \varnothing \text { infinitely often }\right\}=1 \text {. }
$$

For each $n \geq 1$, define $G_{n}=\cup_{k \geq n} F_{k}$, and note that it is an open set in $\partial T$. It is evident from the previous display that

$$
\mathbb{P}\left\{G_{n} \cap U \neq \varnothing \forall n \geq 1\right\}=1 .
$$

Since $m \geq 1$ and $\tau \in \partial T$ were arbitrary, this development shows that with probability one, every $G_{n}$ is an open nowhere dense random subset of $\partial T$. By the Baire category theorem, with probability one, $\cap_{n} G_{n} \neq \varnothing$. This means that almost surely,

$$
\exists \sigma \in \partial T: S_{n(k+1)}^{\sigma}-S_{n(k)}^{\sigma} \geq \sqrt{2 q\left(n_{k+1}-n_{k}\right) f\left(n_{k}\right)} \text {, for infinitely many } k \text {. }
$$

Arguing as we did at the end of our proof of Theorem 2.1 leads us to the following almost-surely valid statement:

$$
\exists \sigma \in \partial T: \limsup _{k \rightarrow \infty} \frac{S_{n(k+1)}^{\sigma}}{\sqrt{2 n_{k+1} f\left(n_{k+1}\right)}} \geq \sup _{\lambda>1} \frac{1}{\sqrt{\varrho(\lambda)}}\left[\sqrt{q\left(1-\frac{1}{\lambda}\right)}-\frac{C_{2.1}}{\sqrt{\lambda}}\right]
$$

see equations (2.6) and (2.7). As we have shown, the right-hand side is positive; moreover, when $T$ is thin, it is equal to $\sqrt{q}$. Since $q \in(0,1)$ is arbitrary, this and Theorem 2.1 together complete our proof. 
Remark 5.2 A close examination of our arguments reveals that spherical symmetry played only a small role in our arguments: It was used to obtain a simple formula for $A_{U}(n)$, and hence $f_{U}(n)$. In fact, the crucial element is embodied in equation (5.1), which is an expression of homogeneity and not spherical symmetry per se. In other words, what is ultimately important is that each ball $U$ is asymptotically as "full" as the tree itself.

We illustrate the validity of this remark by constructing a simple example next.

Example 5.3 Here we construct an example that shows that in Theorem 5.1 spherical symmetry cannot be dropped altogether. In fact, it is possible to generalize this example to prove that the extension of Theorem 5.1 alluded in the previous remark is best possible.

Consider the locally finite rooted tree that is plotted in the figure below. In this tree, all vertices have out-degree one, except those on the "left-most" infinite ray; all of these vertices have out-degree two. It should be recognized that the boundary $\partial T$ of this tree is countable, and yet $A_{n}=\left|\partial T_{n}\right|=n+1$ for all $n \geq 0$. In particular, $T$ is thin, $f(n)=(1+o(1)) \ln n$ as $n \rightarrow \infty$, and $\operatorname{dim}_{\mathcal{L}}(\partial T)=+\infty$.

For any fixed $\sigma \in \partial T$, the following is a consequence of the classical law of the iterated logarithm for random walks: With probability one,

$$
\lim _{n \rightarrow \infty} \frac{\left|S_{n}^{\sigma}\right|}{\sqrt{2 n f(n)}}=0 .
$$

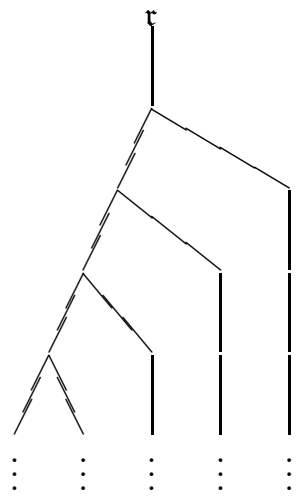

Figure 5.1: A tree with a countable boundary

Since there are only countably many such $\sigma \in \partial T$, the null set in question can be chosen to be independent of $\sigma \in \partial T$, so that

$$
\sup _{\sigma \in \partial T} \lim _{n \rightarrow \infty} \frac{\left|S_{n}^{\sigma}\right|}{\sqrt{2 n f(n)}}=0, \quad \mathbb{P}^{\mathcal{E}} \text {-a.s. }
$$

Thus Theorem 5.1 does not hold for the tree of this example.

\section{Non-Existence of Near-Optimal Rays}

Theorem 5.1 shows that when $T$ is spherically symmetric, the lim sup rate of our law of the iterated logarithm is achieved along some ray. In words, if $T$ is viewed as a decision tree, then there are strategies that infinitely often achieve the best possible return. We now show that the corresponding lim inf behavior can be radically different. 
Theorem 6.1 If $T$ is thin and spherically symmetric, then

$$
\sup _{\sigma \in \partial T} \liminf _{n \rightarrow \infty} \frac{S_{n}^{\sigma}}{\sqrt{2 n f(n)}} \leq 0, \quad \mathbb{P}^{\mathcal{E}} \text {-a.s. }
$$

Proof Fix a positive integer $j_{0}$. Let $\lambda>1$ and for each integer $k \geq j_{0}$, let $n(k)=n_{k}=\left\lfloor\lambda^{k}\right\rfloor$. Let $c \in(0,1)$ be given, and let

$$
G_{k}=\left\{\sigma \in \partial T_{n(k)}: S_{n(j)}^{\sigma}-S_{n(j-1)}^{\sigma} \geq \sqrt{2 c\left(n_{j}-n_{j-1}\right) f\left(n_{j-1}\right)} \quad \forall j_{0} \leq j \leq k\right\} .
$$

Throughout we set $N_{k}=\left|G_{k}\right|$, and will compute $\mathbb{E}\left[N_{k}\right]$, which is the expected number of "good" paths.

For $k \geq 1$, let

$$
p_{k}=\mathbb{P}\left\{S_{n(k+1)}^{\sigma}-S_{n(k)}^{\sigma} \geq \sqrt{2 c\left(n_{k+1}-n_{k}\right) f\left(n_{k}\right)}\right\},
$$

and write

$$
\mathbb{E}\left[N_{k+1}\right]=\sum_{\ell=0}^{\infty} \mathbb{E}\left[N_{k+1} \mid N_{k}=\ell\right] \times \mathbb{P}\left\{N_{k}=\ell\right\} .
$$

If there are $\ell$ good paths in $\partial T_{n(k)}$, then by spherical symmetry, each of them will have $A_{n(k+1)} / A_{n(k)}$ descendants in $\partial T_{n(k+1)}$. Now each of these descendant paths has a $p_{k}$ chance of creating a good path in $\partial T_{n(k+1)}$; thus,

$$
\mathbb{E}\left[N_{k+1} \mid N_{k}=\ell\right]=p_{k} \frac{A_{n(k+1)}}{A_{n(k)}} \ell .
$$

The previous two displays together imply that

$$
\mathbb{E}\left[N_{k+1}\right]=p_{k} \frac{A_{n(k+1)}}{A_{n(k)}} \mathbb{E}\left[N_{k}\right] .
$$

By moderate deviations (Lemma 2.2),

$$
p_{k}=k^{-c+o(1)} A_{n(k)}^{-c+o(1)},
$$

and the thinness of $T$ implies the following:

$$
A_{n(k+1)}=A_{n(k)}^{1+o(1)} .
$$

As a consequence, we obtain

$$
\frac{\mathbb{E}\left[N_{k+1}\right]}{\mathbb{E}\left[N_{k}\right]}=\left(k A_{n(k)}\right)^{-c+o(1)}=o(1) \quad(k \rightarrow \infty),
$$

which shows that as $k \rightarrow \infty, \mathbb{E}\left[N_{k}\right] \rightarrow 0$ exponentially fast. This guarantees that $\sum_{k=1}^{\infty} \mathbb{E}\left[N_{k}\right]<+\infty$, and thanks to the Borel-Cantelli lemma, that $\mathbb{P}\left\{N_{k}=\right.$ 0 eventually $\}=1$. Recalling that $j_{0}$ is arbitrary, we may conclude that

$$
\sup _{\sigma \in \partial T} \liminf _{k \rightarrow \infty} \frac{S_{n(k+1)}^{\sigma}-S_{n(k)}^{\sigma}}{\sqrt{2\left(n_{k+1}-n_{k}\right) f\left(n_{k}\right)}} \leq \sqrt{2 c}, \quad \mathbb{P}^{\mathcal{E}} \text {-a.s. }
$$


Since the value of $c>0$ is immaterial, we can let $c \downarrow 0$ along a rational sequence, and deduce that

$$
\sup _{\sigma \in \partial T} \liminf _{k \rightarrow \infty} \frac{S_{n(k+1)}^{\sigma}-S_{n(k)}^{\sigma}}{\sqrt{2\left(n_{k+1}-n_{k}\right) f\left(n_{k}\right)}} \leq 0, \quad \mathbb{P}^{\mathcal{E}} \text {-a.s. }
$$

Now, as $k \rightarrow \infty$,

$$
n_{k+1}=(\lambda+o(1)) n_{k}, \text { and } f\left(n_{k+1}\right)=(1+o(1)) f\left(n_{k}\right) .
$$

Together with Theorem 2.1 and the preceding display, this yields

$$
\begin{aligned}
\sup _{\sigma \in \partial T} \liminf _{n \rightarrow \infty} \frac{S_{n}^{\sigma}}{\sqrt{2 n f(n)}} & \leq \limsup _{k \rightarrow \infty} \frac{M_{n(k)}}{\sqrt{2\left(n_{k+1}-n_{k}\right) f\left(n_{k}\right)}} \\
& \leq \frac{1}{\sqrt{\lambda-1}}, \quad \mathbb{P}^{\mathcal{E}} \text {-a.s. }
\end{aligned}
$$

This finishes the proof, since $\lambda$ can be chosen arbitrarily large.

\section{A Sketch of Proof of Lemma 2.2}

The lower bound borrows an idea from DE ACOSTA (1983, Lemma 2.4) that is, in turn, from DE Acosta AND Kuelbs (1983, Lemma 3.1). We will sketch this idea presently.

Let $\alpha>0$ and write $S_{n}=V_{1}+\cdots+V_{n}=\sum_{i=1}^{\alpha x_{n}} S_{n, j}$ where $S_{n, 1}, \ldots, S_{n, \alpha x_{n}}$ are i.i.d., each with the same distribution as $S_{n /\left(\alpha x_{n}\right)}$. (This demonstration is a sketch only because we are pretending that $\alpha x_{n}$ is an integer; a similar remark will apply to our proof of the upper bound. Patching up all of this is not hard and the details are left to the interested reader.)

$$
\begin{aligned}
\mathbb{P}\left\{S_{n} \geq \sqrt{n x_{n}}\right\} & =\mathbb{P}\left\{\sum_{j=1}^{\alpha x_{n}} S_{n, j} \geq \sqrt{n x_{n}}\right\} \\
& \geq\left[\mathbb{P}\left\{S_{n, 1} \geq \frac{1}{\alpha} \sqrt{\frac{n}{x_{n}}}\right\}\right]^{\alpha x_{n}} \\
& =\left[\mathbb{P}\left\{S_{n /\left(\alpha x_{n}\right)} \geq \frac{1}{\alpha} \sqrt{\frac{n}{x_{n}}}\right\}\right]^{\alpha x_{n}} .
\end{aligned}
$$

Thus, by the central limit theorem, and writing $g$ for a standard normal variate, we have

$$
\liminf _{n \rightarrow \infty} \frac{1}{x_{n}} \ln \mathbb{P}\left\{S_{n} \geq \sqrt{n x_{n}}\right\} \geq \alpha \ln \mathbb{P}\left\{g \geq \alpha^{-\frac{1}{2}}\right\}, \quad \forall \alpha>0 .
$$

Let $\alpha \rightarrow \infty$ to obtain the lower bound from the standard Gaussian estimate: As $\beta \rightarrow 0, \ln \mathbb{P}\left\{g>\beta^{-1}\right\}=-\left(\frac{1}{2}+o(1)\right) \beta^{-2}$. Notice that this half only requires the existence of two finite moments. 
For the upper bound, we need some finite exponential moments, as we will use a rescaled large deviations argument. Indeed, note that

$$
\begin{aligned}
\mathbb{P}\left\{S_{n} \geq \sqrt{n x_{n}}\right\} & =\mathbb{P}\left\{\sum_{j=1}^{\alpha x_{n}} S_{n, j} \geq \sqrt{n x_{n}}\right\} \\
& =\mathbb{P}\left\{\sum_{j=1}^{\alpha x_{n}} \frac{S_{n, j}}{\sqrt{n / x_{n}}} \geq x_{n}\right\} \\
& \leq\left[\mathbb{E}\left\{\exp \left(\frac{S_{n, j}}{\sqrt{n / x_{n}}}\right)\right\}\right]^{\alpha x_{n}} e^{-x_{n}},
\end{aligned}
$$

where all of the above expressions are finite for sufficiently large $n$. Thus, for all $n$ large enough,

$$
\frac{1}{x_{n}} \ln \mathbb{P}\left\{S_{n} \geq \sqrt{n x_{n}}\right\} \leq \alpha \ln \mathbb{E}\left\{\exp \left(\frac{S_{n /\left(\alpha x_{n}\right)}}{\sqrt{n / x_{n}}}\right)\right\}-1 .
$$

By the central limit theorem, $\lim \sup _{n \rightarrow \infty} \frac{1}{x_{n}} \ln \mathbb{P}\left\{S_{n} \geq \sqrt{n x_{n}}\right\} \leq-\frac{1}{2}$. provided that $\alpha>\lambda_{0}^{-2}$. Thus far we have shown that

$$
\mathbb{P}\left\{S_{n} \geq \sqrt{n x_{n}}\right\}=\exp \left\{-\frac{1+o(1)}{2} x_{n}\right\}, \quad(n \rightarrow \infty) .
$$

This finishes our proof, since we can replace $S_{n}$ by $\max _{1 \leq j \leq n} S_{j}$ by Ottaviani's maximal inequality; see CHUNG (1974, p. 120).

\section{References}

[1] DE Acosta, A. (1983). A new proof of the Hartman-Wintner law of the iterated logarithm, Ann. Prob., 11, 270-276.

[2] De Acosta, A. And J. Kuelbs (1981). Some new results on the cluster set $\mathbf{C}\left(\left\{S_{n} / a_{n}\right\}\right)$ and the LIL, Ann. Prob., 11, 102-122.

[3] Aldous, D. (1992). Greedy search on a tree with random edge-weights, Comb., Prob. \& Computing, 1, 281-293.

[4] Benjamini, I. And Y. Peres (1994). Tree-indexed random walks and firstpassage percolation, Prob. Th. Rel. Fields, 98, 91-112.

[5] Biggins, J. D. (1977). Chernoff's theorem in the branching random walk, J. Appl. Prob., 14, 630-636.

[6] Chung, K. L. (1974). A First Course in Probability Theory, Second Edition, Academic Press, New York.

[7] Dembo, A. And O. Zeitouni (1998). Large Deviations Techniques and Applications, Second Edition. Springer, Berlin. 
[8] Dubins, L. And D. Freedman (1967). Random Distribution Functions, Proc. Fifth Berkeley Symp. on Math. Stat. and Prob., 2(1), 183-214. (L. Le Cam and J. Neyman, Ed.'s)

[9] Esary, J. D. , F. Proschan, and D. W. Walkup (1967). Association of random variables, with applications, Ann. Math. Stat., 38, 1466-1474.

[10] Hall, P. And C. C. Heyde (1980). Martingale Limit Theory and its Applications, Academic Press, New York.

[11] Hammersley, J. M. (1974). Postulates for subadditive processes. Ann. Prob., 2, 652-680.

[12] Khoshnevisan, D., Y. Peres and Yimin Xiao (1999). Limsup random fractals, Elec. J. Prob., 5(4), 1-24

[13] Kingman, J. F. C. (1975). The birth problem for an age-dependent branch process, Ann. Prob., 12, 341-345.

[14] Lyons, R. (1990). Random walks and percolation on trees, Ann. Prob., 18, 931-958.

[15] Lyons, R. (1992). Random walks, capacity and percolation on trees, Ann. Prob., 20, 2043-2088.

[16] Lyons, R. And R. Pemantle (1992). Random walks in a random environment and first-passage percolation on trees, Ann. Prob., 20, 125-136.

[17] Peres, Y. (1999). Probability on Trees: An Introductory Climb, Saint-Flour Lectures, 1997, Lect. Notes in Math., to appear in Springer-Verlag.

[18] Virág, B. (2001). Fast graphs for the random walker, Prob. Th. Rel. Fields (to appear).

Davar Khoshnevisan. The University of Utah, Department of Mathematics, Salt Lake City, UT 84112-0090, U. S. A.

davar@math.utah.edu

http://www.math. utah.edu/ davar

Thomas M. Lewis. Furman University. Department of Mathematics. Greenville, SC 29613, U. S. A.

tlewis@math.furman .edu

http://math.furman.edu/ ${ }^{\sim}$ llewis 